\title{
Gestión de la Diversidad entre Pequeños Comercios de Barcelona. Análisis de una Experiencia de Mediación Comunitaria Intercultural
}

\section{Diversity Management Among Small Shops in Barcelona: A Case of Community Intercultural Mediation}

\author{
Mercé Zegrí, Josep M. Navarro y Míkel Aramburu \\ Fundació Desenvolupament Comunitari.
}

\begin{abstract}
Resumen. La instalación de pequeños comercios regentados por personas de origen extranjero presenta una serie de dificultades y retos en cuanto a su encaje en ciertos barrios y territorios, cuestiones que se han intentado abordar desde la mediación intercultural. Mediante este tipo de intervención se pretende incidir en las relaciones y la comunicación entre comerciantes haciendo visibles sus intereses comunes, así como en sus estructuras asociativas, de manera que éstas reconozcan y se adapten a las nuevas realidades. En este artículo se analiza un proceso de intervención de mediación comunitaria intercultural en el ámbito del pequeño comercio llevado a cabo en un barrio de Barcelona. En primer lugar, se exponen los resultados más significativos de la fase de diagnóstico, en cuanto a discursos y relaciones de los agentes del territorio y el análisis del "conflicto" y, en segundo lugar, se detalla la intervención de mediación, realizada en el marco de un grupo de comerciantes que llamamos "grupo promotor del comercio". Finalmente, se realizan algunas valoraciones generales respecto al impacto de este tipo de procesos en el territorio así como a su continuidad y sostenibilidad, teniendo en cuenta su alto potencial participativo y transformador.

Palabras clave: comerciantes, comercio extranjero, desarrollo comunitario, mediación intercultural, conflicto.
\end{abstract}

Summary. The setting up of small shops by foreign people involves a number of difficulties and challenges with respect to how it fits in certain districts. These issues have been tackled from the view of intercultural mediation. This kind of intervention focuses on relationships and communication among shopkeepers by highlighting their common interests. Intervention also includes making associative networks acknowledge and adapt to new realities. This paper describes a process of intervention focused on community intercultural mediation carried out in small shops in a district of Barcelona. First, most significant results of the diagnosis stage are shown concerning the speech and relationships of main agents along with "conflict" analysis. Then a detailed mediation intervention is described that was carried out with a group of shopkeepers called "shop promoting group". Finally, bearing in mind their high participative and transformational potential, some general appraisals are made with respect to the impact of this type of processes and their continuity and sustainability.

Key words: shopkeepers, Shops kept by foreign people, Community Development, Intercultural Mediation, Conflict. 
Los comercios regentados por inmigrados no comunitarios están transformando el paisaje comercial de Barcelona. Estos pequeños comercios de base familiar empezaron a proliferar durante la década de los noventa, principalmente en los barrios del casco viejo de la ciudad como el Raval y Casc Antic, en el distrito de Ciutat Vella. A partir del 2000 empiezan a verse comercios de inmigrantes en muchas otras zonas de la ciudad donde la población inmigrada no comunitaria ha empezado a asentarse más tardíamente.

El texto que presentamos aquí analiza la tensa relación entre comerciantes autóctonos e inmigrados en el barrio de Poble Sec, en el distrito de SantMontjuic de Barcelona, y, más concretamente, se centra en una experiencia de mediación comunitaria entre ambos colectivos, que ha permitido abrir nuevos espacios de encuentro y horizontes de futuro compartido.

El comercio inmigrante ha devenido en la retórica de los pequeños comerciantes tradicionales el chivo expiatorio de la crisis del propio pequeño comercio de proximidad, crisis que, no es necesario decirlo, tiene unas causas estructurales mucho más amplias. A ello hay que añadir la fragilidad del tejido asociativo comercial de estos barrios, desvertebrado por la debilidad de su estructura organizativa y la desorientación en que les ha sumido la desaparición de los viejos comercios y la llegada de los nuevos, ante los cuales predomina el desconocimiento, la desconfianza y la falta de comunicación.

Hablamos de mediación comunitaria porque, de alguna manera, persigue reconstruir los lazos y vínculos relacionales de la comunidad, desplazando las percepciones de "peligrosidad social" que en ocasiones se generan entre ciertos comerciantes y ciertos sectores de la población. Los comercios barriales y de proximidad tienen un claro papel en la vertebración comunitaria, pues son "nodos" que entrelazan muchas personas diversas que residen en el mismo territorio. Asimismo, son escenarios donde se generan (y reproducen) discursos, juicios y prejuicios sobre el territorio y los grupos que lo habitan. El pequeño y mediano comercio de barrio tiene, en definitiva, una importancia significativa en el desarrollo de actitudes de acogida o de rechazo hacia los nuevos vecinos.
La intervención mediadora realizada en el barrio del Poble Sec entre febrero de 2004 y mayo de 2005 estaba enmarcada dentro de un servicio de mediación comunitaria intercultural puesto en marcha por la concejalía de Bienestar Social del Ayuntamiento de Barcelona ${ }^{2}$. Las intervenciones de mediación realizadas $^{3}$ venían normalmente motivadas por la detección de un cierto "mal clima" o "mal ambiente" en torno a la actividad de los comercios extranjeros. Por parte del Ayuntamiento, la intervención mediadora se inscribía dentro de una estrategia más amplia de revitalización de zonas vulnerables de la ciudad, no únicamente como una medida de promoción económica, sino también como una forma de vertebración social y territorial de estos barrios, en los cuales los pequeños comercios de proximidad juegan un papel fundamental.

Las grandes líneas operativas de la mediación comunitaria intercultural son, por un lado, visibilizar y reconocer la diversidad (biográfica, cultural, social y de visiones sobre el entorno) y, por otro, hacer aflorar los intereses, miradas e inquietudes comunes. Se trata, en definitiva, de incorporar las diferencias en un proyecto común de desarrollo local. La intervención de mediación es un proceso que dura aproximadamente un año y que tiene dos fases diferenciadas pero estrechamente interrelacionadas.

Durante los cuatro primeros meses se realiza un diagnóstico, un trabajo de campo que nos sirve para escuchar lo que los diferentes actores tienen que decir y proponer sobre la actividad comercial y su entorno urbano, así como para identificar los agentes sociales clave del territorio y reconstruir y analizar sus relaciones. Con ello obtenemos algunas claves substantivas y relacionales para orientar el proceso de mediación.

En la segunda fase, propiamente de mediación, se invita a una serie de comerciantes representativos de la diversidad del tejido comercial a participar en espacios de conocimiento y diálogo creados al efecto, donde se intenta poner las condiciones para un

\footnotetext{
2 Aunque el servicio es una iniciativa municipal, entre 2002-2007 fue gestionado por un equipo técnico de la Fundación Desenvolupament Comunitari, que trabajaba en coordinación con los técnicos/as municipales de servicios personales de cada distrito.

${ }^{3}$ Además del Poble Sec se intervino en otras calles del Raval y Casc Antic de Ciutat Vella, así como en el barrio de Roquetas (distrito Nou Barris).
} 
nuevo proyecto de dinamización comercial de la zona en el que todos los actores se sientan representados.

\section{El discurso de los comerciantes}

El barrio de Poble Sec (15.000 personas) está encajonado entre la montaña de Montjuïc, sobre cuyas faldas se encabalga, y la avenida Paralelo, la emblemática arteria donde antaño se ubicaban los principales teatros y cabarets de la ciudad. Se trata de un barrio popular donde la inmigración extracomunitaria ha contrarrestado un largo proceso de declive social y demográfico. La pérdida de la población más activa en términos productivos y reproductivos había llevado a un envejecimiento y pauperización de la población, todo lo cual había agudizado en este territorio la crisis del pequeño comercio tradicional. Independientemente de otro tipo de consideraciones que puedan realizarse, no hay duda de que la llegada de inmigrantes extracomunitarios y la apertura de tiendas por parte de éstos ha supuesto para ésta como para otras zonas de la ciudad, un proceso innegable de rejuvenecimiento demográfico y revitalización comercial.

Sin embargo, en el Poble Sec, como en los otros casos en que hemos trabajado, la revitalización que supone el comercio extranjero no siempre es reconocida por la población autóctona, y especialmente por los comerciantes "de toda la vida", entre quienes predomina cierta hostilidad hacia los comerciantes de origen inmigrante, que son vistos como una amenaza severa e irreversible para su identidad territorial y son objeto de severos reproches.

De hecho, la demanda de mediación venía motivada porque una serie de asociaciones de vecinos y comerciantes se estaba movilizando contra los comercios inmigrantes. Las sospechas de las ilegalidades e irregularidades más diversas habían sido recogidas, como si fuesen hechos comprobados, en un informe que se llegó a filtrar a la prensa ${ }^{4}$.

4 Comissió de Seguretat Ciutadana del Poble Sec: "Estudi sobre locals comercials de Queviures, Locutoris i Bars en el territori del Poble Sec" (noviembre de 2003). Informaciones del informe aparecieron en el diario local de tirada gratuita "20 Minutos" (23 de octubre de 2004) además de en alguna publicación de barrio.
Con este trasfondo, nos propusimos una aproximación a los comerciantes del barrio para comprender sus visiones y posiciones en toda su singularidad y diversidad. Y es que los comerciantes autóctonos no forman un colectivo homogéneo. A grandes rasgos, deberíamos distinguir al menos entre dos tipos de comerciantes autóctonos que configuran perfiles característicos en cuanto a su arraigo en la zona y discurso comercial. Por un lado está el comercio tradicional o "de toda la vida", según la categorización nativa, y por otro lado un comercio más joven y moderno y con una identidad cosmopolita.

El comerciante "tradicional" no suele estar muy lejos de la jubilación y probablemente cuando eso ocurra cerrará y traspasará su negocio por falta de continuidad familiar. Se trata de personas que llevan muchos años regentando un negocio familiar, orientado a una clientela de barrio, y que afrontan las vicisitudes del pequeño comercio de proximidad con un apoyo familiar menguante.

Por otra parte, el comerciante autóctono "moderno" ha establecido su negocio recientemente y se presenta a sí mismo con una identidad cosmopolita y abierta a los cambios. Sus negocios pueden pertenecer a sectores tradicionales como bares, restaurantes y tiendas de ropa, pero dotados de algún marchamo especial, o bien a actividades "innovadoras" (tatuajes y piercing, tiendas de dietética...) que se dirigen a un público territorialmente diverso, de dentro y de fuera del barrio.

Para los comerciantes tradicionales que enfrentan las transformaciones de vecindario, de una clientela fiel que va menguando, así como los cambios en los hábitos de consumo y la competencia de las medianas superficies que van asentándose en sus barrios, el comerciante inmigrante suele servir de chivo expiatorio de su inseguridad y malestar.

La rumorología es el caldo de cultivo de donde se nutren las constantes sospechas sobre las supuestas irregularidades e ilegalidades que infringen los comerciantes inmigrados (impago de impuestos, falta de licencias, irregularidades en la venta de productos, usar las tiendas como "tapaderas" de actividades delictivas, incumplimiento de la ley de horarios comerciales, explotación a sus trabajadores, etc.), insinuando a veces un trato de favor por parte de la Administración. También surgen otras críticas, 
como la "guetificación" de sus actividades comerciales, que favorecerían las relaciones monoculturales, la ocupación incívica del espacio público por parte de la clientela de bares y restaurantes, su oferta comercial "homogénea" o su imagen "cutre".

En cambio, para los comerciantes "modernos", aunque pueden criticar al comercio extranjero por la homogeneidad de su oferta o por la escasa promoción de "productos culturales", este tipo de comercio supone para ellos una diversidad étnica que se integra a su proyecto comercial como un valor positivo, exótico, y que encaja con su modelo de barrio, en proceso de progresiva semejanza a las cosmopolitas Londres o París. Estos jóvenes comerciantes también critican al comercio tradicional, porque no renueva su oferta ni dinamiza actividades culturales.

Mención aparte merecen las asociaciones de comerciantes. Dirigidas por comerciantes autóctonos "de toda la vida", estas asociaciones suelen mantener una actividad mortecina, siendo mínimo, cuando no nulo, el conocimiento que de éstas tienen los nuevos comerciantes. En general, en el Poble Sec, todos los comerciantes coincidían en que estas asociaciones no funcionaban. Para los comerciantes modernos el problema era que las asociaciones no se habían renovado con gente joven ni impulsaban actividades innovadoras más allá de la lotería y la instalación de las luces de Navidad en la calle. Para los comerciantes tradicionales el problema fundamental era la incidencia negativa que sobre el tejido comercial había tenido la llegada de inmigrantes, sin ignorar por eso la falta de motivación y participación del resto de comerciantes.

Las charlas con los comerciantes de proximidad, ya fueran autóctonos o extranjeros, ponían en evidencia cierta ética común propia del pequeño comercio (el ahorro y las pequeñas inversiones por encima de los gastos suntuosos, el sacrificio y apoyo familiar por encima de las estrategias individuales) que posteriormente, en el proceso de mediación, se intentaría hacer visible.

Respecto a la caracterización y discurso de los comerciantes extranjeros del Poble Sec, no podemos establecer muchas diferencias internas. La práctica totalidad de los comerciantes extranjeros habían llegado en fechas recientes. Se trata de negocios incipientes, cuyos dueños combinan con otros trabajos, concentrando el grueso de sus relaciones en el seno de su comunidad étnica. Recién llegados y con un escaso conocimiento del entorno, expresan un discurso simple y escueto, más centrado en la marcha de sus negocios y en su kafkiana relación con la Administración, plagada de impedimentos y lentos y complejos trámites burocráticos. Por todo ello, están siempre sobre-ocupados, con otras prioridades y con escaso tiempo para implicarse en la mejora de un tejido asociativo comercial que normalmente desconocen. Aún así, en el Poble Sec valoraban muy positivamente el barrio y el vecindario, y se mostraban optimistas respecto al futuro.

No obstante, también entre los comerciantes inmigrantes encontramos personas con un perfil más "moderno". Originarios de África Subsahariana, de países de América Latina o de Marruecos, regentan bares musicales, teterías o tiendas de artesanía típica de sus países, y comparten con los comerciantes autóctonos modernos el interés por conseguir una clientela amplia, de dentro y de fuera del barrio, y por dinamizar actividades culturales.

Así, la polarización entre comerciantes autóctonos y comerciantes alóctonos, a los que se suponía intereses e identidades contrapuestos, va haciéndose más compleja, desdoblándose, a través del análisis del discurso, en nuevas polarizaciones que van apareciendo (en torno a cuestiones como el ocio nocturno, la estética comercial o la implicación en actividades culturales) que confrontan a autóctonos con autóctonos, a inmigrantes con inmigrantes, y que tienden puentes insospechados entre autóctonos y extranjeros.

\section{La caracterización del conflicto}

La información obtenida mediante el diagnóstico debe aportarnos los elementos necesarios para diseñar una estrategia de mediación con los comerciantes. En la mediación de conflictos suele distinguirse entre dos componentes o dimensiones diferentes que contribuyen a la producción del conflicto: el interés y la identidad.

En el primer caso, la acción o el comportamiento de una parte va en detrimento de los intereses de la otra. Por ejemplo, en nuestro caso los comerciantes autóctonos pueden acusar la competencia de unos 
horarios comerciales más dilatados, o como vecinos y usuarios del espacio público pueden sentirse molestos por la juerga nocturna de los clientes de los bares dominicanos. En el segundo caso, lo que genera el conflicto no es tanto la acción o el comportamiento de una de las partes sino su identidad. Lo que molesta del otro no es lo que él hace sino lo que es, y sobretodo que esté aquí. En el primer caso, lo que chocan son intereses divergentes, en el segundo son las identidades las que son incompatibles. Esta distinción es puramente analítica. En la práctica, ambas dimensiones no se dan en estado puro, sino que se interrelacionan; p.e., el ruido de los bares dominicanos molesta por ruidoso y por dominicano. Es difícil imaginar un conflicto de intereses en el que la identidad de las partes no juegue papel alguno, pero aun siendo difíciles de separar con nitidez, ambas dimensiones (interés e identidad) pueden tener un peso diferente en la generación y reproducción del conflicto. Conocer este peso diferencial es fundamental para la radiografía del conflicto y para la estrategia de mediación.

En el Poble Sec, el análisis nos indica que el conflicto entre los comerciantes es básicamente unidireccional: son los comerciantes autóctonos los que se sienten en conflicto con los extranjeros. Por otro lado, aunque los comercios de extranjeros puedan mermar algo los intereses comerciales de los comerciantes autóctonos, la competencia es sólo parcial ya que en gran parte se trata de una oferta complementaria, y aunque operen en el mismo sector se dirigen a segmentos diferentes del mercado. Asimismo, las acusaciones de trato de favor por parte de la administración no tienen ninguna base. En ningún caso puede achacarse a los comercios extranjeros la crisis del pequeño comercio de proximidad de base familiar. Los factores interpretativos que pueden ayudarnos a comprender la actitud hostil de los comercios autóctonos ante los recién llegados es el miedo al desclasamiento y la inquietud ante los cambios, aspectos que se ven alimentados por el desconocimiento interpersonal y la falta de referentes compartidos ante la ausencia de un pasado y un proyecto en común.

Esto nos lleva a orientar las estrategias de mediación a la búsqueda de un espacio donde se puedan conocer, donde a partir del diálogo pueda emerger un proyecto común. Es decir, más que en las identidades strictu sensu, se pretende incidir en los procesos de identificación a partir de propuestas y de proyectos construidos conjuntamente. Una estrategia basada en alcanzar acuerdos entre las partes (modelo de Harvard) sería más adecuada para un conflicto de intereses ${ }^{5}$. En cambio, ante un conflicto de este tipo de lo que se trata principalmente es de hacer emerger, de visibilizar los puntos en común, es decir, canalizar de forma más intensa los puntos de vista que comparten sin saberlo y, sobretodo, los intereses comunes que, como pequeños comerciantes, tienen.

\section{La devolución del diagnóstico}

La devolución del diagnóstico es el primer paso de la intervención propiamente mediadora. Una vez acabado el diagnóstico y presentado al distrito, se convoca a todas las personas que han sido entrevistadas y a los técnicos/as del distrito referentes para presentar los resultados y acordar el plan de trabajo de la mediación. Se trata de un momento de especial relevancia en el proceso de mediación, ya que es la primera vez en que la mediación se escenifica en un acto colectivo. Hasta ahora, los actores sólo habían tenido conocimiento de la intervención mediadora a través de las entrevistas realizadas para el diagnóstico. Ahora, los actores del territorio implicados en el "problema" se ven como colectivo, formando parte de un mismo proceso en el que los mediadores resultan reconocidos y legitimados. La presencia de los técnicos del distrito avala el proceso (lanza el mensaje de que va "de verdad") pero se intenta que la reunión no se convierta en una constante interpelación a la administración, ni que ésta "adoctrine" o lanze consignas, o pronuncie discursos sin concretar. Los mediadores son los que guían las intervenciones e invitan a los técnicos/as (ubicados en un lugar marginal de la escena) a responder preguntas o aclarar cuestiones puntuales.

En el Poble Sec asistieron unas 50 personas (comerciantes y miembros entrevistados de entidades del barrio y técnicos/as del distrito).

${ }^{5}$ Sobre los modelos de mediación y su aplicación a los conflictos interculturales, véase Giménez 2001. 
La devolución del diagnóstico se realiza en clave mediadora. En este sentido, se intenta lograr que los diferentes actores se identifiquen y reconozcan en el diagnóstico y a la vez que puedan ver sus opiniones contrastadas con las del resto. Al mismo tiempo se introduce el propio discurso de los mediadores, basado en sus observaciones e interpretaciones. Con ello se intenta introducir una mirada externa que aporte nuevos elementos que cuestionen y enriquezcan la visión del problema. En el marco de las teorías transformativa y circular narrativa, este podría considerarse el primer paso de la mediación, en el cual, la contrastación de los diferentes discursos, incluyéndose el del mediador/a, intenta avanzar en el "reconocimiento" y la "revalorización" de sí mismo y de los otros en la resolución del "conflicto".

En el Poble Sec la propuesta de plan de trabajo se centró en el comercio y en su promoción, a partir de la elaboración de un proyecto de dinamización comercial del barrio. El proyecto sería elaborado por un grupo de comerciantes diversos, mediante unas sesiones de trabajo, el apoyo del equipo mediador y la colaboración del Ayuntamiento. La propuesta fue aceptada, quedando implícito el compromiso de los asistentes de apoyar el proceso.

\section{La creación del grupo promotor del comercio}

La intervención de mediación comercial se realizó en el marco de lo que llamamos "grupo promotor del comercio", un grupo de entre 7 y 14 comerciantes creado por el equipo mediador. El proceso de mediación se orienta a que el grupo promotor vaya consolidándose como equipo de trabajo, asumiendo su responsabilidad y compromiso en la resolución de los problemas del comercio en el barrio.

Los criterios de selección de los participantes son fundamentalmente tres: 1) que sean representativos de la diversidad existente en el territorio en cuanto a tiempo de estancia, tipo de actividad, discurso, nacionalidad y vinculación con las asociaciones de comerciantes, 2) que estén motivados para participar en el proceso o al menos que se detecte cierta potencialidad de motivación, y 3 ) que tengan un elevado grado de conectividad en sus respectivas redes sociales comerciales.
La selección de los componentes del grupo promotor (que llamamos "agentes clave") es una de las principales decisiones del proceso de mediación, ya que de su grado de acierto depende en gran medida el éxito de todo el proceso.

El grupo promotor implica un desplazamiento del centro gravitatorio de la cohesión y participación de los comerciantes del territorio. La intervención mediadora no se realiza en el marco de las asociaciones de comerciantes que, desprestigiadas y atrapadas en rutinas de funcionamiento poco constructivas, no serían un terreno neutral para la mediación. En cambio, el grupo promotor del comercio proporciona un terreno más neutral, creado por un agente externo que, en esta fase, ya está legitimado como mediador. No obstante, las asociaciones de comerciantes no se marginan. Bien al contrario, se insiste especialmente en atraer la presencia de miembros destacados de las juntas de las asociaciones. En todo caso, en la selección de los componentes del grupo promotor es importante que ningún "sector" pueda sentirse marginado o excluido del proceso.

En el Poble Sec el grupo promotor lo formaron 7 comerciantes, un grupo compuesto de perfiles diversos: dos representantes del tejido comercial tradicional del barrio, uno con un discurso más excluyente hacia los comerciantes extranjeros y otro con una postura más moderada y parcialmente integradora; dos personas representativas del comercio llamado "moderno" o "cosmopolita" y que a su vez se identificaban con dos estilos diferentes de comercio existente en el barrio (uno más alternativo y representativo del ocio nocturno y el otro más elitista, el de la restauración de alta calidad); dos comerciantes pakistaníes con cierta antigüedad en el barrio ( 3 años) y con una estrategia de ampliación y diversificación de sus negocios (pequeños supermercados, locutorios y restaurantes); y, finalmente, la propietaria dominicana de un bar restaurante, integrada a su vez en un grupo informal de propietarios de bares dominicanos del distrito Sants-Montjuïc, que quería constituirse en asociación para dialogar con los vecinos/as y la Administración sobre los problemas que se generaban alrededor de sus negocios.

Los miembros del grupo promotor configuraban un grupo heterogéneo y diverso en cuanto a los diversos perfiles, intereses, discursos e identidades 
en juego, guardando un cierto equilibrio respecto a algunas de las principales variables sociológicas (origen, género y edad). La argamasa que les unía era un relativo arraigo en el barrio y un cierto interés mostrado por mejorar el barrio así como en implicarse en ello.

\section{La mediación en el "grupo promotor"}

El "grupo promotor de comercio" trabaja durante una serie de sesiones en la elaboración de un proyecto de dinamización comercial. A demanda del propio grupo o a propuesta del equipo mediador, también se organizan sesiones dedicadas a la asesoría o información sobre temas de interés diversos (planes urbanísticos previstos en la zona, asociacionismo, información sobre ayudas institucionales al comercio, intercambio con otras asociaciones de comerciantes...), para lo cual se busca un experto/a o técnico/a municipal. El equipo mediador conduce las sesiones, aportando metodologías que posibiliten la participación de todos y ayudando a la concreción de las ideas.

La elaboración del proyecto de dinamización comercial que se va desarrollando en diferentes sesiones tiene tres fases distintivas: a) diagnóstico colectivo del comercio del barrio; b) definición de objetivos del proyecto; y c) concreción de las actividades del proyecto.

El desarrollo de las sesiones supone un avance en tres direcciones: respecto al proyecto, respecto al (re)conocimiento de los comerciantes, y respecto a la consolidación del grupo. En el camino de estrechar relaciones y generar confianza entre unos y otros, la elaboración del proyecto en todas sus fases ayuda a visibilizar los problemas e intereses compartidos poniendo en común las memorias de cada uno, construyendo aquello que la teoría circular narrativa llamaría una "nueva historia" compartida. En este sentido, la fase de realización del diagnóstico colectivo incidiría en la "historia" pasada y presente, mientras que en las fases de definición de objetivos y actividades se construiría un "proyecto" compartido.

Sin espacio para poder detallar aquí todo el proceso de trabajo con el "grupo promotor" del Poble
Sec, vamos a centrarnos en algunos momentos críticos o de inflexión en el proceso de mediación.

Comenzando por el diagnóstico colectivo que hizo el grupo sobre el comercio y su barrio, uno de los temas que más atención suscitó entre los comerciantes fue el comportamiento de algunos jóvenes en el espacio público (escándalos nocturnos, actos vandálicos, hurtos, consumo de droga,...). Mientras se exponían estos problemas, uno de los comerciantes pakistaníes expresó su malestar por un hecho relacionado que le afectaba diariamente y perjudicaba gravemente su negocio. Cada día, al final de la tarde, un grupo de jóvenes del barrio aparcaba sus motos frente a su supermercado y se quedaba allí charlando y fumando porros. Como la acera era estrecha y el establecimiento estaba abierto a la calle, el olor del hachís quemado entraba en la tienda. Más allá de la molestia, el comerciante expresaba su preocupación porque sabía de los rumores que acusaban a los locutorios pakistaníes, como el que él mismo tenía en esa misma calle, de vender droga a los jóvenes. El comerciante reclamó a la técnica del distrito que participaba en la sesión una solución urgente del problema. Este fue un momento de inflexión importante en el proceso de mediación ya que para muchos de los comerciantes presentes (en particular para la presidenta de la asociación de comerciantes de la misma calle) supuso un cambio en la mirada hacia esa persona con la que por primera vez se evidenciaba una visión compartida sobre un tema tan candente como el del denominado "incivismo juvenil", tan vinculado por otra parte a la inmigración extranjera. En el mismo sentido, también fue importante que la comerciante de origen dominicano expusiera el trabajo que estaba realizando en el marco del grupo de restauradores dominicanos del distrito, visibilizando así la voluntad de este colectivo de abordar los problemas que se generaban alrededor de sus negocios y sus "escandalosas clientelas".

Asimismo, otro de los comerciantes pakistaníes, animado por el equipo mediador, reconstruyó su trayectoria laboral, que en cierta forma era bastante representativa del colectivo, contribuyendo a que los nativos comprendieran y en cierta forma empatizaran con las vicisitudes de los comerciantes extranjeros, a través de la historia personal de uno de ellos, 
contada en primera persona, en la que podían encontrar elementos en común con su propia experiencia.

En las últimas sesiones de trabajo se hace siempre evidente la necesidad de crear o reactivar una asociación de comerciantes del barrio como forma de poder llevar a cabo el proyecto de dinamización comercial. Los miembros del grupo promotor son los que normalmente se presentan como candidatos para formar parte de la junta de la nueva asociación. La nueva asociación, dotada de una junta multicultural, renace con la idea de llegar a todos los comerciantes del barrio.

En el transcurso del proceso, la diversidad del grupo promotor pasa de problema a fortaleza, dando confianza y seguridad al grupo, en la medida en que éste reconoce en su diversidad la propia diversidad del barrio, pero una diversidad que funciona, que dialoga, que llega a acuerdos, que construye conjuntamente un nuevo proyecto.

\section{Reflexiones finales}

Quisiéramos concluir con algunas valoraciones críticas del proceso de mediación, valoraciones que tienen una validez que va más allá del caso concreto del Poble Sec.

Aunque, formalmente, la mediación se realiza entre actores de la comunidad (de la sociedad civil) que por una u otra razón están en conflicto, la Administración y especialmente la Administración Municipal resulta directamente concernida en la medida en que es la responsable de proveer y gestionar aspectos fundamentales del bienestar de los ciudadanos. La administración debe saber mantenerse en posición de ser interpelada y de responder en consecuencia. Cumplir con este rol no suele resultar fácil, y el equipo mediador debe tener la habilidad de mantener al Ayuntamiento en su papel al mismo tiempo que depende orgánicamente de él. Ésta no es una tarea sencilla y necesita ser manejada con cuidado, pero sólo si la independencia técnica del equipo se hace patente puede éste tener credibilidad ante los actores sociales y el proceso de mediación tener éxito.

Asimismo, la mediación y el consecuente proceso de empoderamiento de los actores sociales implica- dos, genera dinámicas con un alto potencial participativo y transformador, sobretodo en la medida en que propicia la puesta en marcha de dinámicas de transversalidad entre áreas de la administración municipal como vía pública y seguridad, comercio y promoción económica, servicios personales o urbanismo. En este sentido, y aunque la transversalidad nunca es fácil, hay cuestiones que surgen en las dinámicas mediadoras y que no siempre encuentran eco en la política urbanística y que redundarían en la mejora del diseño de espacios públicos, en una mayor identificación con las reformas urbanísticas y en la generación de mayor consenso sobre la forma de hacer ciudad.

Nuestra preocupación se centra actualmente en cómo mantener y realimentar la participación de los actores, que justamente debe garantizar la sostenibilidad real del proceso iniciado con la mediación. Por este motivo nos encontramos en una fase de reflexión sobre la metodología empleada en cada fase para tratar de garantizar una mayor estabilidad participativa de los actores, sobre todo de aquellos con una dinámica de movilidad espacial a escala global más que de inserción territorial concreta a la vieja usanza. Cómo conciliar un proyecto de mediación comunitaria fuertemente territorializado con estrategias, identidades y lealtades de actores que trascienden lugares concretos, es una de los principales desafíos que enfrenta un proyecto de este tipo.

Por último, más allá del éxito a corto plazo, al equipo mediador le gustaría provocar cambios más profundos, de mediano y largo alcance, sobre aspectos culturales, ideológicos y de actitudes ante la diversidad y el desarrollo comunitario. Aunque la intervención ha ayudado a dar un paso en ese sentido, no sabemos en qué grado han cambiado los prejuicios y estereotipos. Pero al menos éstos no han impedido iniciar una dinámica de diálogo y colaboración.

Todavía desconocemos cuál será la incidencia de estas renovadas asociaciones, y del pequeño comercio en general, en la dinámica de sus territorios en el marco de profundas transformaciones económicas y sociales. En todo caso, del proceso de mediación surge, en un primer momento, un tejido comercial empoderado, adaptado a la realidad diversa de su barrio, y con una energía renovada para afrontar los 
retos que plantean las dinámicas socieconómicas en curso para los comercios barriales y los barrios interculturales.

\section{Bibliografía}

Aramburu, M. (2002). "Los comercios de inmigrantes extranjeros en Barcelona y la recomposición del 'inmigrante' como categoría social”, en Scripta Nova. Revista electrónica de geografía y ciencias sociales, $\mathrm{n}^{\circ} 108$.

Arjona, A. \& Checa, J. C . (2004), "El empresariado étnico como alternativa a la discriminación laboral: la situación en Almería", ponencia presentada en el VIII Congreso Español de Sociología, Alicante.

Beltrán, J. \& Sáiz, A. (2001) Comunidades asiáticas en España. Barcelona, Fundació CiIDOB, Barcelona.

Giménez, C. (2001) "Modelos de mediación y su aplicación en mediación intercultural", Migraciones, $\mathrm{n}^{\circ} 10$, Madrid.
Moreras, J. (2002): Les activitats comercials dels estrangers a Ciutat Vella, Barcelona, Fundació CIDOB.

Oso, L. \& Ribas, N. (2004): "Empresariado étnico y género: dominicanas y marroquíes en Madrid y Barcelona", ponencia presentada en el $I V$ Congreso Español de la Inmigración en España, Girona.

Sole, C. \& Parella, S. (2005) Negocios étnicos. Los comercios de los inmigrantes no comunitarios en Cataluña. Barcelona, Fundació CIDOB.

Zegrí, M., Navarro, J. M. \& Aramburu, M. (2006): "El encaje de los comercios extranjeros en el tejido comercial de acogida. Análisis de una experiencia de mediación comunitaria en Barcelona", Migraciones, $\mathrm{n}^{\circ}$ 20, Madrid

Zegri, M., Vidaurrazaga, E. (2008): “Associacionisme comercial, un mitjà de cohesió social i de cooperació intercultural", Diversitat cultural $i$ globalització: nous reptes per al moviment associatiu (coord: J. Pascual y E. Rovira, Fundació Desenvolupament Comunitari), Ajuntament de Barcelona.

Manuscrito recibido: 08/01/2009

Revisión recibida: 15/02/2009

Manuscrito aceptado: 23/02/2009 